\title{
Effet de différentes méthodes d'échantillonnage sur les valeurs d'indices biotiques de la qualité de l'eau du cours inférieur d'un fleuve méditerranéen : le Tibre
}

\author{
A.G. Solimini 1 \\ P. Gulia ${ }^{1}$ \\ M. Monfrinotti ${ }^{1}$ \\ G. Carchini ${ }^{1}$
}

Mots-clés: substrats artificiels, macroinvertébrés, Tibre.

Cette note se propose de confronter l'aptitude de deux indices biotiques à la détermination de la qualité de l'environnement du cours inférieur du Tibre et d'évaluer si et comment la méthode d'échantillonnage peut influencer la valeur finale de ces indices. Une des méthodes met en œuvre un filet troubleau utilisé pendant 3', tandis que les autres sont basées sur l'utilisation de substrats artificiels immergés à chaque station pendant toute la durée de l'étude. Nos résultats semblent démontrer une sensibilité différente pour les deux indices biotiques dans la description des changements de la qualité de l'environnement et une influence significative de la technique d'échantillonnage sur la détermination de la qualité des eaux obtenue avec les indices biotiques.

Effect of different sampling methods on the biotic indices values of the water quality in the lower part of the mediterranean River Tiber

Keywords : artificial substrata, macroinvertebrates, Tiber river.

This paper aims to test the performance of two different biotic indices and the effect of four different sampling methods in assessing the water quality of the final tract of the River Tiber. One sampling methods involves use of a hand net for 3 minutes, the others use articial substrata left in water for all the study period. Our results showed a different performance of the two biotic indices and a significant effect of the sampling method on scores of the biotic indices.

\section{Introduction}

Au cours des dernières années, les objectifs de l'évaluation des ressources aquatiques, partis de la protection des standards de qualité des eaux destinées à différents usages (eau potable - eau d'irrigation agricole) ont été la préservation ou la restauration de l'intégrité biologique des écosystèmes. L'approche traditionnelle, basée sur la détermination des variables chimiques et physiques, s'est révélée de moins en moins satisfaisante, à cause de la grande variabilité de ces mesures (tant

1. Laboratorio di Ecologia Sperimentale ed Acquacoltura, Dipartimento di Biologia, Università di Roma "Tor Vergata", Via della Ricerca Scientifica, 00133 Roma, Italia. dans l'espace que dans le temps) et parce qu'elle n'offre qu'un aperçu partiel du problème. L'évaluation biologique - usage systématique des organismes ou de leur réponse au milieu pour la détermination de la qualité de ce milieu - offre quelques caractéristiques intéressantes, comme la possibilité d'intégrer les variations temporelles de la qualité du milieu et de donner une mesure directe des effets des perturbations, qui sont complémentaires aux données de l'évaluation chimique et physique.

A ce propos, les macroinvertébrés sont le groupe le plus fréquemment utilisé en raison d'une série de caractéristiques qui en rendent l'usage plus aisé (voir Rosenberg \& Resh 1993). Une des approches possibles avec ces organismes prévoit le calcul d'indices biotiques qui concentrent en un seul chiffre l'information qui découle de la composition de la communauté. En 
Europe on a proposé et utilisé plus de 30 indices biotiques (voir pour une liste récente De Pauw et al. 1992) qui diffèrent entre eux principalement par le niveau taxonomique requis et la façon de calculer le score final. Comme les différents indices, bien que recommandés pour un usage général, sont liés aux conditions qui en ont déterminé la génèse, il est important que ces dernières soient examinées de façon critique. Cet aspect a cependant été fréquemment sous-évalué et l'usage des différents indices est souvent plutôt subjective.

En région méditerranéenne, les zones montagneuses prédominent sur les plaines, où se concentrent les populations humaines et leurs activités agricoles et industrielles. En conséquence, de nombreuses grandes villes sont situées auprès des cours inférieurs de fleuves et rivières qui reçoivent, ipso facto, les effluents urbains, industriels et ceux provenant d'une agriculture intensive. D'où la grande importance pratique de la mise au point de méthodes efficaces de détermination de la qualité de l'environnement de la partie inférieure des cours d'eau. Malheureusement, plusieurs des indices biotiques proposés en Europe mettent en œuvre un protocole d'échantillonnage adapté aux torrents ou aux cours d'eau peu profonds. Par contre, les cours inférieurs des fleuves (zones potamiques) présentent des conditions qui ne permettent pas d'utiliser les techniques habituelles d'échantillonnage (filet de Surber, échantillonneur de Hess). La benne elle même, instrument communément utilisé en faciés lentique, ne permet pas un échantillonnage efficace de microhabitats comme la végétation ou des substratums à granulométrie supérieure à quelques millimètres où les macroinvertébrés sont très fréquents. Pour de telles zones potamiques, de nombreux manuels traitant de l'application d'indices biotiques préconisent l'utilisation de substrats artificiels (Hellawell 1986, Ghetti 1986). Cependant, aucune méthode d'échantillonnage standard n'est proposée, et le choix du matériel est souvent laissé à l'initiative du chercheur.

Cette note se propose de comparer la pertinence de deux indices biotiques différents, à la détermination de la qualité de l'environnement du cours inférieur du Tibre et d'évaluer l'influence de la méthode d'échantillonnage sur la valeur finale des indices. Les résultats obtenus avec les différents indices et les différentes méthodes d'échantillonnage sont confrontés soit avec un ensemble de variables chimiques et physiques utiles aux fins pour la détermination de la qualité des eaux, soit avec une description de la communauté macrobenthique effectuée avec des méthodes statistiques multivariées, sans imposer un poids différentiel aux différents taxa.

\section{Zone d'étude, matériel et méthodes}

\subsection{Zone d'étude}

La zone d'étude est constituée par les 60 derniers km du cours du Tibre, qui présentent des caractéristiques homogènes de profondeur, de courant, de type de sédiment et de typologie des rivages. Ce tronçon de rivière, dans une large mesure urbanisé par la traversée de la ville de Rome (environ 3 millions d'habitants), présente un degré de pollution croissante vers l'embouchure par l'arrivée successive des effluents des stations d'épuration des égouts et d'autres rejets non traités.

\subsection{Méthodes d'échantillonnage des macroinverté- brés}

Un total de 10 stations d'échantillonnage a été retenu (lettres $S$, Fig. 1), dont la dernière est située juste en amont de l'embouchure dans la mer Tyrrhénienne. A chaque site les invertébrés macrobenthiques ont été récoltés saisonnièrement entre mai 1993 et mars 1994 selon 4 méthodes d'échantillonnage différentes. Une méthode met en œuvre un filet troubleau manœuvré à la main (méthode 1) ; avec ce filet nous avons échantillonné tous les microhabitats visibles pendant une durée de trois minutes. Les trois autres méthodes se basent sur l'utilisation de substrats artificiels immergés à chaque station pendant toute la durée de l'étude. Le premier type de substrat était constitué par un fagot de minces branches de noisetier enveloppé dans un filet métallique à mailles hexagonales de $5 \mathrm{~cm}$ déterminant une structure cylindrique de $20 \mathrm{~cm}$ de diamètre, $50 \mathrm{~cm}$ de longueur et $2 \mathrm{Kg}$ de poids frais. Un de ces fagots était ancré au fond (méthode 2 : substrat organique de fond) tandis qu' un autre était attaché à un flotteur (méthode 3 : substrat organique flottant). Le deuxième type de substrat était construit selon le même principe mais les branches étaient en plastique et formaient une structure de $15 \mathrm{~cm}$ de diamètre, $60 \mathrm{~cm}$ de longueur et une superficie totale de $67,5 \mathrm{dm}^{2}$. Cette structure (méthode 4 : substrat inorganique) était censer simuler une plante aquatique commune le long du fleuve (Myriophyllum sp.). Suivant les observations publiées sur le temps nécessaire pour stabiliser une communauté de macroinvertébrés sur des substrats artificiels (Meier et al. 1979, De Pauw et al. 1986), tous les substrats sont restés immergés pendant plus de deux mois avant chaque date d'échantillonnage (y compris la première).

A échéance saisonnière, les invertébrés récoltés, fixés dans de l'alcool à $75^{\circ}$, étaient déterminés au niveau taxonomique approprié au calcul des indices biotiques et comptés. Les variables physiques et chimiques ont été mesurées pour chaque saison par l'Ad- 


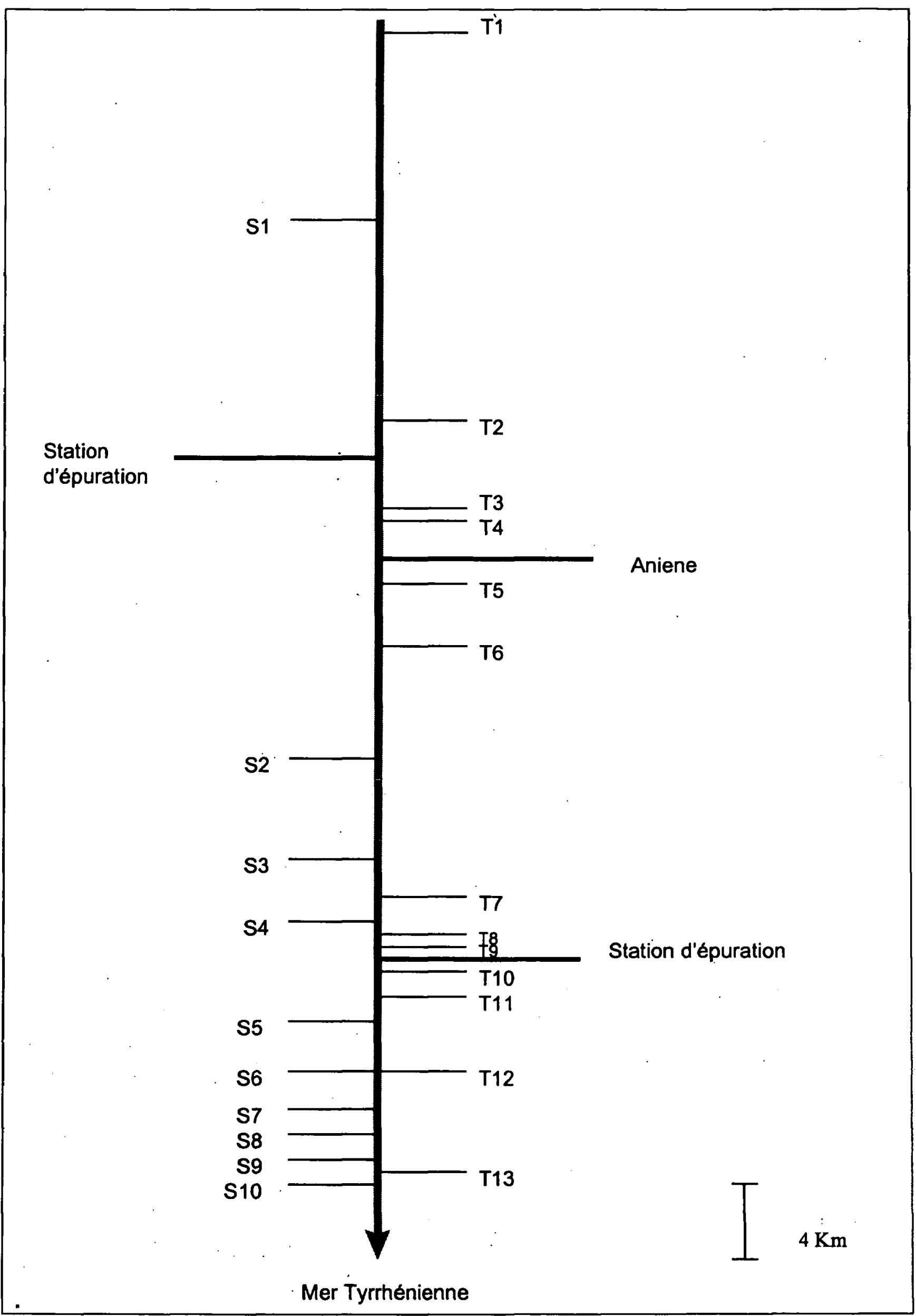

Fig. 1. Carte du cours inférieur du Tibre avec l'emplacement des stations d'échantillonnage pour les paramètres chimiques et physiques (lettres $T$ ) et pour les macroinvertébrés (lettres $S$ ). Une bonne partie de la zone d'étude se trouve en secteur fortement urbanisée (Rome).

Fig. 1. Study area with sampling sites for chemical - physical variables (letter T) and macroinvertebrates (letter S). A large part of the area studied lies in a heavily urbanised sector of Rome. 
ministration Communale pour l'Energie et l'Environnement de Rome (ACEA 1994 : lettres T, Fig. 1).

\subsection{Analyse des données et indices biotiques}

L'absence de traitement statistique pour vérifier les résultats a souvent été l'une des critiques aux méthodes biologiques pour l'évaluation de la qualité des eaux (Karr 1994). Dans cette étude on s'est donc efforcé d'aller au delà des simples aspects descriptifs en privilégiant une approche analytique capable de prendre en compte les aspects quantitatifs.

Pour l'analyse des données nous avons suivi les étapes suivantes : (i) classement multivarié des sites d'échantillonnage sur la base des variables chimiques et physiques et sur celle de la composition de la communauté de macroinvertébrés ; (ii) contrôle de la cohérence des scores des indices biotiques avec les classements du point (i) ; (iii) contrôle de la sensibilité des indices à la technique d'échantillonnage utilisée.

Les deux indices biotiques utilisés sont : 1) le BMWP' - Biological Monitoring Working Party — dans la version adaptée à la réalité méditerranéenne par Alba-Tercedor \& Sanchez-Ortega (1988) ; 2) l'EBI - Extended Biotic Index - dans la version adaptée à la situation italienne et révisée récemment par Ghetti (1995). Suite aux révisions de Ghetti (1995), le niveau de détermination taxonomique exigé par les deux indices est actuellement très semblable (la famille, dans la quasi-totalité des cas) tandis que le système d'évaluation reste différent. Le classement des sites d'échantillonnage a été obtenu grâce à une analyse en composantes principales (ACP) à partir d'une matrice de 13 sites x 19 variables chimiques et physiques enregistrées par l'ACEA (valeurs ponctuelles ; voir ACEA 1994). L'organisation des données biologiques a été obtenue avec une analyse en coordonnées principales (Gower 1966), à partir d'une matrice des distances entre les sites calculée avec la formüle de Sokal \& Michener (1958), sur la base de la présence des 41 taxa de macroinvertébrés dans les 10 sites d'échantillonnage, durant la période d'étude. La similarité obtenue à partir des indices biotiques entre les groupes de stations formés d'après l'analyse précédente a été calculée en pourcentages selon Resh \& Jackson (1993). La significativité statistique a été évaluée avec le test de Kruskal-Wallis.

\section{Résultats}

\subsection{Classement des sites sur la base des variables chimiques, physiques et biologiques}

Le classement des sites effectué avec une ACP-sur les valeurs des variables physico-chimiques montre la présence de 3 groupes homogènes de stations, en progressant de l'amont vers l'aval (Fig. 2). Ces groupes sont séparés par les principaux rejets polluants sur le cours du Tibre étudié, représentés par la rivière Aniene, entre le premier et le deuxième groupe, et par le rejet de la station d'épuration de Rome Sud, entre le deuxième et le troisième. Un classement des stations, effectué avec une analyse en coordonnés principales sur la faune de macroinvertébrés (Tableau 1) montre le même regroupement des stations, séparées par les mêmes rejets (Fig. 3).

\subsection{Contrôle de la cohérence entre les scores des in- dices biotiques et le classement des stations}

Les scores obtenu avec les deux indices biotiques sont montrés sur le tableau 2. Pour examiner l'aptitude des deux indices biotiques à diversifier les trois groupes (A correspondant à la station $\mathrm{S} 1$; B à $\mathrm{S} 2, \mathrm{~S} 3$ et $\mathrm{S} 4$; $\mathrm{C}$ à toutes les autres stations) obtenus par l'analyse multivariée, nous avons calculé le pourcentage de similarité entre les trois groupes sur la base des scores des indices biotiques obtenus seulement avec la méthode 1 (Tableau 3). Les deux indices sont à même d'apprécier les variations de la qualité de l'environnement en passant d'un groupe à l'autre, mais le BMWP' est plus sensible que l'EBI. En outre, les différences entre les groupes, sur la base des scores des BMWP', sont toutes statistiquement significatives. Au contraire, l'EBI a uniquement montré une différence significative entre les scores des groupes A et C.

\subsection{Contrôle de la sensibilité des indices biotiques à la technique d'échantillonnage utilisée}

Les scores obtenus avec les deux indices biotiques ont été testés par rapport aux différentes techniques d'échantillonnage utilisées. Seul le BMWP' a montré une différence significative entre les scores obtenus en échantillonnant avec le filet a main et avec 2 des 3 substrats artificiels (Tableau 4). Aucune variation n'a au contraire été constatée avec l'EBI. Les résultats sont partiellement faussés par l'absence de score pour $\mathrm{S} 1$ avec les méthodes 2,3 , et 4 .

\section{Discussion}

Le cours inférieur du Tibre a été l'objet de nombreuses études sur la dégradation générale de l'environnement dû aux écoulements polluants importants de la ville de Rome (Zoppini \& Puddu 1995, Battegazzore et al. 1995, Solimini et al. 1997).

Nos résultats semblent démontrer : 1) une sensibilité différente des_deux indices biotiques pour la description des changements de la qualité de l'environnement ; 
Tableau 1. Faune des macroinvertébrés récoltée aux différentes stations du Tibre ( $1=$ présence $; 0=$ absence).

Table 1. Macroinvertebrate fauna collected at different sampling sites along the River Tiber $(1=$ presence $; 0=a b s e n c e)$.

\begin{tabular}{|c|c|c|c|c|c|c|c|c|c|c|}
\hline \multirow[t]{2}{*}{ Taxon } & \multicolumn{10}{|c|}{ Sites } \\
\hline & $\mathrm{S} 1$ & $\mathbf{S 2}$ & S3 & S4 & S5 & S6 & S7 & S8 & S9 & S10 \\
\hline Dugesia $\mathrm{sp}$ & 1 & 1 & 0 & 0 & 0 & 0 & 1 & 0 & 0 & 1 \\
\hline Tubifex sp & 1 & 1 & 1 & 1 & 1 & 1 & 1 & 1 & 1 & 1 \\
\hline Limnodrilus sp & 1 & 1 & 1 & 1 & 1 & 1 & 1 & 1 & 1 & 1 \\
\hline Psammoryctides $\mathrm{sp}$ & 0 & 0 & 1 & 0 & 1 & 0 & 0 & 0 & 0 & 1 \\
\hline Naididae & 0 & 1 & 0 & 1 & 1 & 0 & 1 & 0 & 1 & 1 \\
\hline Enchytraeidae & 0 & 0 & 0 & 1 & 0 & 0 & 0 & 0 & 0 & 0 \\
\hline Lumbricidae & 1 & 0 & 0 & 0 & 0 & 0 & 0 & 0 & 0 & 0 \\
\hline Haplotaxidae & 1 & 0 & 0 & 0 & 0 & 0 & 0 & 0 & 0 & 0 \\
\hline Dina lineata & 1 & 0 & 0 & 0 & 0 & 0 & 0 & 0 & 0 & 0 \\
\hline Erpobdella testacea & 0 & 0 & 1 & 0 & 1 & 1 & 0 & 1 & 0 & 0 \\
\hline Helobdella stagnalis & 0 & 1 & 0 & 0 & 0 & 0 & 0 & 0 & 0 & 0 \\
\hline Piscicola geometra & 0 & 0 & 1 & 0 & 0 & 0 & 0 & 0 & 1 & 0 \\
\hline Bithynia tentaculata & 1 & 1 & 1 & 1 & 1 & 1 & 1 & 1 & 1 & 1 \\
\hline Physa acuta & 1 & 1 & 1 & 1 & 1 & 1 & 1 & 1 & 1 & 1 \\
\hline Potamopyrgus antipodarum & 1 & 0 & 0 & 0 & 0 & 0 & 0 & 0 & 0 & 0 \\
\hline Theodoxus fluviatilis & 1 & 1 & 0 & 0 & 1 & 0 & 0 & 0 & 0 & 0 \\
\hline Pisidium sp & 1 & 0 & 0 & 0 & 0 & 1 & 0 & 0 & 1 & 0 \\
\hline Sphaerium sp & 1 & 0 & 0 & 0 & 0 & 0 & 0 & 0 & 0 & 0 \\
\hline Asellus aquaticus & 1 & 0 & 0 & 1 & 0 & 0 & 0 & 0 & 0 & 1 \\
\hline Proasellus coxalis & 1 & 1 & 1 & 1 & 1 & 1 & 1 & 1 & 1 & 1 \\
\hline Palaemonetes antennarius & 1 & 0 & 0 & 0 & 0 & 0 & 1 & 1 & 1 & 1 \\
\hline Atyaephyra desmaresti & 0 & 1 & 0 & 1 & 0 & 0 & 0 & 0 & 0 & 0 \\
\hline Echinogammarus veneris & 1 & 1 & 1 & 1 & 1 & 0 & 1 & 0 & 1 & 1 \\
\hline Caenis sp & 1 & 0 & 0 & 0 & 0 & 0 & 0 & 0 & 0 & 0 \\
\hline Cercion lindeni & 1 & 1 & 1 & 1 & 0 & 1 & 1 & 0 & 0 & 0 \\
\hline Erythromma viridulum & 1 & 0 & 1 & 1 & 1 & 1 & 1 & 0 & 0 & 0 \\
\hline Ischnura elegans & 1 & 1 & 1 & 1 & 0 & 0 & 0 & 0 & 0 & 0 \\
\hline Platycnemis pennipes & 1 & 0 & 0 & 0 & 0 & 0 & 0 & 0 & 0 & 0 \\
\hline Coenagrium puella & 1 & 0 & 0 & 0 & 0 & 0 & 0 & 0 & 0 & 0 \\
\hline Anax sp & 1 & 0 & 0 & 0 & 0 & 0 & 0 & 0 & 0 & 0 \\
\hline Corixa sp & 1 & 0 & 0 & 0 & 0 & 1 & 0 & 0 & 0 & 1 \\
\hline Micronecta sp & 1 & 1 & 0 & 0 & 0 & 0 & 0 & 1 & 1 & 1 \\
\hline Aphelocheirus aestivalis & 0 & 0 & 0 & 0 & 0 & 0 & 0 & 0 & 0 & 1 \\
\hline Sisyridae & 0 & 0 & 1 & 0 & 0 & 0 & 0 & 0 & 0 & 0 \\
\hline Haliplidae & 1 & 0 & 0 & 0 & 0 & 0 & 0 & 0 & 0 & 0 \\
\hline Polycentropodidae & 1 & 0 & 0 & 0 & 0 & 0 & 0 & 0 & 0 & 0 \\
\hline Ceratopogonidae & 0 & 1 & 0 & 1 & 0 & 0 & 0 & 0 & 0 & 0 \\
\hline Chironomidae & 1 & 1 & 1 & 1 & 1 & 1 & 1 & 1 & 1 & 1 \\
\hline Culicidae & 0 & 0 & 0 & 0 & 1 & 0 & 0 & 0 & 0 & 0 \\
\hline Psycodidae & 1 & 0 & 1 & 1 & 0 & 0 & 0 & 0 & 0 & 0 \\
\hline Tipulidae & 0 & 1 & 0 & 0 & 0 & 0 & 0 & 0 & 0 & 0 \\
\hline
\end{tabular}




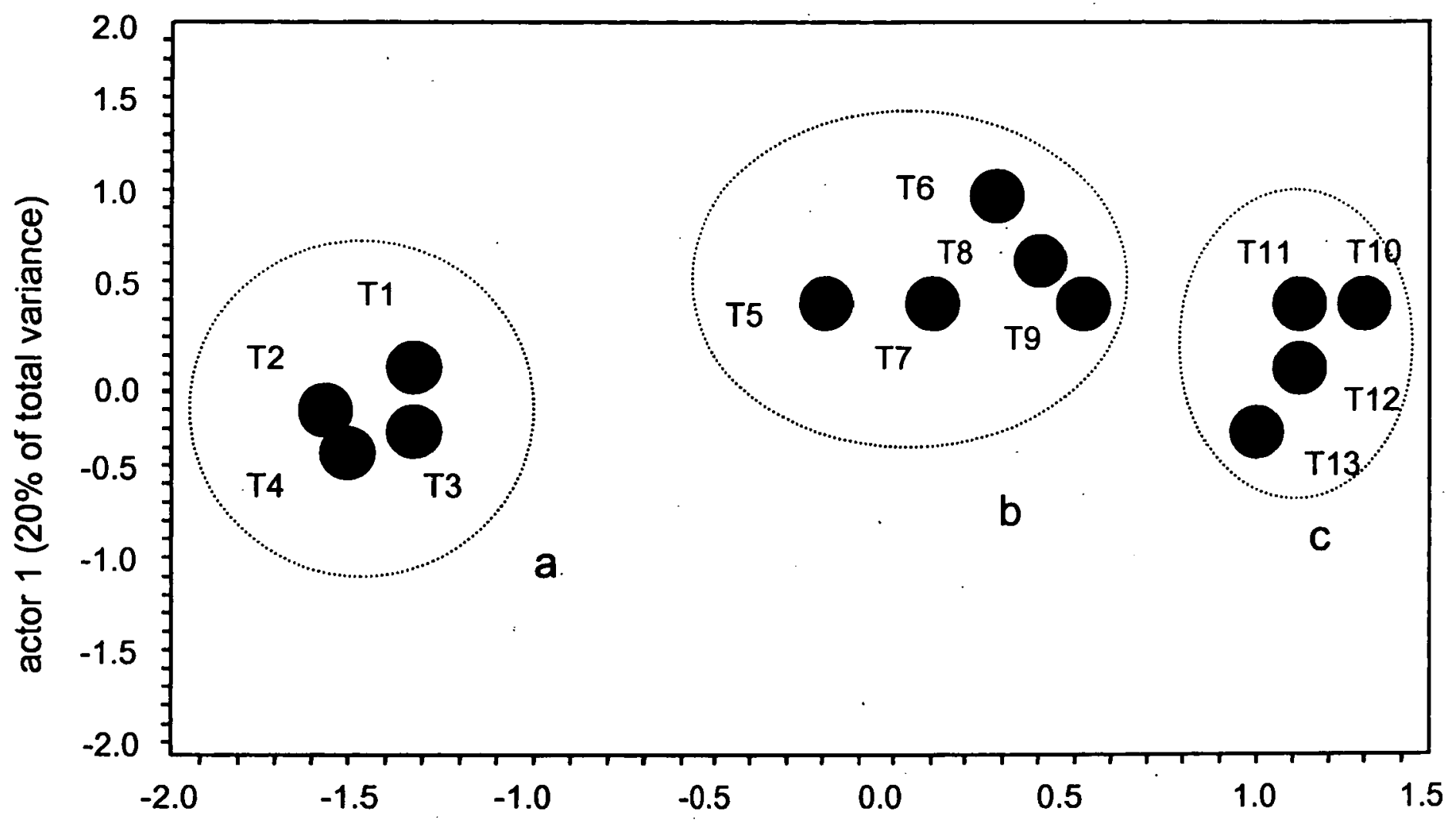

Fig. 2. Classement des sites d'échantillonnage obtenu par une analyse en composantes principales sur la base des variables chimiques et physiques mesurées mensuellement par l'ACEA.

Fig. 2. Ordination of sampling site using principal components analysis on monthly chemical - physical variables by ACEA.

Tableau 2. Scores moyens des 4 valeurs saisonnières des indices biotiques BMWP' et EBI obtenus avec les 4 différentes méthodes d'échantillonnage $(1=$ filet troubleau; 2 = substrat organique de fond; $3=$ substrat organique flottant; $4=$ substrat inorganique).

Table 2. Mean scores $(\mathrm{N}=4$ seasonal samples) of biotic indexes BMWP' and EBI with the different sampling methods ( 1 = hand net, 2 = organic bottom substrate; 3 = organic floating substrate; $4=$ inorganic substrate).

\begin{tabular}{lllllllll}
\hline & \multicolumn{3}{c}{ BMWP' Méthode } & \multicolumn{5}{c}{ EBI Méthode } \\
\hline Site & 1 & 2 & 3 & 4 & 1 & 2 & 3 & 4 \\
\hline S1 & 45.7 & - & - & - & 6.3 & - & - & - \\
S2 & 26.7 & 33.2 & 30 & - & 5 & 5 & 4.7 & - \\
S3 & 20.5 & 33.5 & 28 & 17.5 & 4.5 & 4.5 & 4.7 & 4.5 \\
S4 & 20.5 & 33.5 & 28 & 17.5 & 4.5 & 4.5 & 5 & 4 \\
S5 & 23 & 15.5 & 19.8 & 16.7 & 4 & 4 & 3.7 & 4 \\
S6 & 12 & 16.5 & 23.7 & 22.3 & 3.2 & 3.7 & 4.2 & 4 \\
S7 & 15.2 & 20.7 & 19.7 & 20 & 4.2 & 4 & 3.7 & 4.5 \\
S8 & 8.5 & 22 & 16.5 & 24.5 & 4 & 4.5 & 3.5 & 4.2 \\
S9 & 13.2 & 18.7 & 21.7 & 19 & 4.7 & 4 & 4.2 & 4.5 \\
S10 & 15.7 & 17.2 & -20.5 & 16.5 & -4.7 & 4.2 & 4.7 & $4.7 \ldots$ \\
\hline
\end{tabular}




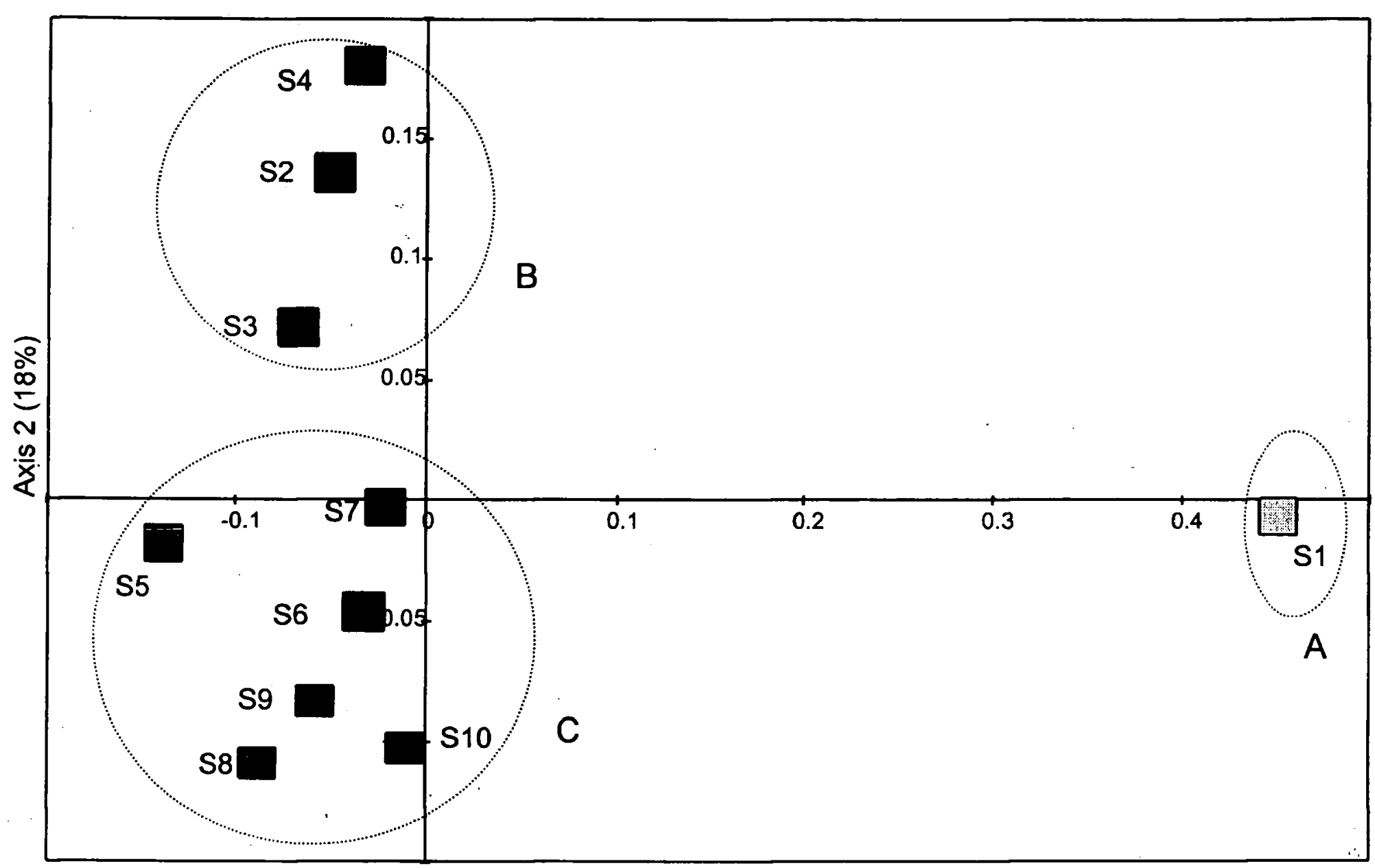

Axis $1(46 \%)$

Fig. 3. Classement des sites d'échantillonnage obtenu par une analyse en coordonnées principales sur la base de la composition des communautés macrobenthiques.

Fig. 3. Ordination of sampling site using principal coordinate analysis on the composition of the macroinvertebrate community.

2) une influence significative de la technique d'échantillonnage sur la détermination de la qualité des eaux obtenue avec ces deux indices biotiques. En particulier, le BMWP' correspond mieux aux résultats de l'analyse multivariée conduite tant sur les données chimiques et physiques que biologiques. L'EBI, dans la version employée dans cette étude (Ghetti 1995), est moins sensible en présence de taux élevés de pollution et croissants. En ce qui concerne l'effet de la méthode d'échantillonnage sur les valeurs d'indices biotiques, le BMWP' semble être plus influençable que l'EBI. Ces différences concernent principalement l'utilisation du troubleau et des substrats artificiels, ces derniers donnant les moins bonnes valeurs. Ce problème peut être dû aux caractéristiques du dernier tronçon du Tibre, où la profondeur moyenne de $10 \mathrm{~m}$, les turbulences élévées et la végétation très rare rendent l'utilisation du troubleau inefficace. Par contre, les différences entre les valeurs obtenues avec les différents substrats artificiels sont moindres. De Pauw et al. (1986) ont également trouvé que la nature des substrats n'était pas un facteur crucial pour la colonisation par les macroinvertébrés, bien qu'ils aient utilisé un type de substrat différent. Dans le Tibre, nous avons observé que les différents substrats étaient rapidement recouverts d'une fine couche de détritus, de telle manière que le type de matériau utilisé, au moins pendant quelques mois, n'est probablement pas important pour la colonisation par les macroinvertébrés.

\section{Conclusion}

Les meilleurs résultats du BMWP' suggèrent la nécessité d'utiliser plus fréquemment cet indice dans les études d'évaluation biologique de la qualité des eaux courantes profondes. Cependant, l'indice BMWP' doit être mieux adapté à la faune fluviale italienne car la liste de référence exclue certaines familles de macroinvertébrés. De plus, dans la'zone potamique des fleuves, le choix d'une méthode d'échantillonnage particulière paraît crucial pour les études d'évaluation biologique. 
Tableau 3. Contrôle de la cohérence des indices biotiques des trois groupes de stations obtenus avec l'analyse multivariée. Le pourcentage de similarité a été calculé d'après Resh \& Jackson (1993) ; en italiques, résultats des tests de KruskalWallis (n.s. = non significatif).

Table 3. Performance of biotic indices in assessing differences of environmental quality between the 3 groups resulting from the multivariate analysis. The percentage of similarity is calculated as in Resh \& Jackson (1993) and tested with Kruskal-Wallis non parametric test.

\begin{tabular}{llll}
\hline Indices & $\mathrm{A}-\mathrm{B}$ & $\mathrm{A}-\mathrm{C}$ & $\mathrm{B}-\mathrm{C}$ \\
\hline BMWP' & 47.99 & 29.74 & 61.97 \\
& $<0.05$ & $<0.05$ & $<0.05$ \\
EBI & 71.05 & 66.00 & 93.00 \\
& n.s. & $<0.05$ & n.s. \\
\hline
\end{tabular}

Tableau 4. Evaluation de la sensibilité des deux indices à la technique d'échantillonnage utilisée. Résultats du test Kruskal-Wallis (n.s.= non significatif) dans les comparaisons entre les scores des deux indices obtenus avec les 4 méthodes d'échantillonnage ( 1 = filet troubleau; 2 = substrat organique de fond; 3 = substrat organique flottant; $4=$ substrat inorganique).

Table 4. Effect of the sampling method on the biotic index scores tested with the Kruskall-Wallis non parametric test. ( 1 = hand net, 2 = organic bottom substrate; 3 = organic floating substrate; 4 = inorganic substrate).

\begin{tabular}{|c|c|c|c|c|c|c|c|c|}
\hline \multicolumn{5}{|c|}{ BMWP' Méthode } & \multicolumn{4}{|c|}{ EBI Méthode } \\
\hline & 1 & 2 & 3 & 4 & 1 & 2 & 3 & 4 \\
\hline \multicolumn{9}{|l|}{1} \\
\hline 2 & $<0.05$ & & & & n.s. & & & \\
\hline 3 & $<0.05$ & n.s. & & & n.s. & n.s. & & \\
\hline 4 & n.s & n.s & n.s & & n.s & n.s & n.s & \\
\hline
\end{tabular}

Dans notre étude, l'approche biologique s'est révélée un outil intéressant pour l'évaluation de la qualité des eaux des cours terminaux de fleuves en pays méditerranéen. Cependant, il semble que le développement d'un protocole commun pour les pays méditerranéens soit nécessaire pour rendre pleinement comparables les résultats. Les éléments fondamentaux d'un tel protocole devront porter d'une part, sur le choix de l'indice biotique qui doit être applicable aux différentes conditions rencontrées et d'autre part, sur le choix des techniques d'échantillonnage et sur le développement d'un procédé d'analyse des données efficace.

\section{Remerciements}

Nous remercions le MURST pour sa contribution financière (programme : "Tassonomia e autoecologia della fauna dulcacquicola e dei suoli"), N. Falchi et A. Queyras pour la traduction du manuscrit en français et un lecteur anonyme pour la révision critique du manuscrit.

\section{Travaux cités}

ACEA, Azienda Comunale Energia \& Ambiente, 1994. - Controllo della qualità del fiume Tevere. Rapporto. ACEA, Roma : 122 p.

Alba-Tercedor J. \& Sanchez-Ortega A. 1988. — Un metodo rapido y simple para evaluar la calidad biologica de las aguas corrientes basado en el de Hellawell (1978). Limnetica, 4: 51-56.

Battegazzore M., Guzzini A., Pagnotta R. \& Marchetti R. 1995. The importance of investigatory and analytical techniques in biological water - quality investigations. In Harper D.M. et Ferguson A.J.D. (eds). The ecological basis for river management. John Wiley \& Sons, New York, USA : 193-209.

De Pauw N., Roels D. \& Fontoura A.P. 1986. - Use of artificial substrates for standardized sampling of macroinvertebrates in the assessment of water quality by the Belgian biotic Index. Hydrobiologia, 133 : 237-258.

De Pauw N., Ghetti P.F., Manzini P. \& Spaggiari R. 1992. - Biological assessment methods for running water. In : Newman P.J., Piavaux M.A. \& Sweeting R.A. (eds.). River water quality ecological assessment and control. Commission of the European Communities, Bruxelles : 217-249. 
Ghetti P.F., 1986. — I macroinvertebrati nell'analisi dei corsi d'acqua. Indice Biotico : E.B.I. modificato. Manuale di applicazione. Provincia Autonoma di Trento, Trento, Italy : $111 \mathrm{p}$.

Ghetti P.F. 1995. - Indice biotico esteso (I.B.E.), metodi di analisi per le acque correnti. Notiziario dei metodi analitici, CNR-IRSA : 31 p.

Gower J.C. 1966. - Some distance properties of latent root and vector methods used in multivariate analysis. Biometrika, 53 : 325-338.

Hellawell J.M. 1986. - Biological indicators of freshwater pollution and environmental management. Elsevier Applied Science, London, UK : $546 \mathrm{p}$.

Karr J.R. 1994. - Biological monitoring : challenges for the future. En : Loeb S.L. \& Spacie A. (eds.). Biological Monitoring of Aquatic systems. Lewis Publisher : 353-373.

Meier P.J., Penrose D.L. \& Polak L. 1979. - The rate of colonization by macro-invertebrates on artificial substrate samplers. Freshwat. Biol., 9 : 381-392.
Resh V.H. \& Jackson J.K. 1993. - Rapid assessment approaches to biomonitoring using benthic macroinvertebrates. In : Rosenberg D.M. \& Resh V.H. (eds). Freshwater biomonitoring and benthic macroinvertebrates. Chapman \& Hall, New York : 195-233.

Rosenberg D.M. \& Resh V.H. 1993. - Introduction to freshwater biomonitoring and benthic macroinvertebrates. In : Rosenberg D.M. \& Resh V.H. (eds). Freshwater biomonitoring and benthic macroinvertebrates. Chapman \& Hall, New York : 1-9.

Sokal R.R. \& Michener C.D. 1958. - A statistical method for evaluating systematic relationships. University of Kansas, Scientific Bullettin, $1: 2-18$

Solimini A.G., Tarallo G.A. \& Carchini G. 1997. - Life history and species composition of the damselfly assemblage along the urban tract of a river in central Italy. Hydrobiologia, 356:21-32.

Zoppini A. \& Puddu A. 1995. - Il perifiton come indicatore di alterazioni ambientali. Confronto di risultati ottenuti dallo studio di due sistemi lotici : il Tevere ed il Po. Atti Soc. ital. E., 16 : 483-485. 